Application of fast sensors to microscope mode spatial imaging mass spectrometry

This article has been downloaded from IOPscience. Please scroll down to see the full text article.

2011 JINST 6 C01044

(http://iopscience.iop.org/1748-0221/6/01/C01044)

View the table of contents for this issue, or go to the journal homepage for more

Download details:

IP Address: 129.67.117.235

The article was downloaded on 11/07/2013 at 11:39

Please note that terms and conditions apply. 
$12^{\text {th }}$ INTERNATIONAL WORKSHOP ON RADIATION IMAGING DETECTORS,

JULY $11^{\text {th }}-15^{\text {th }} 2010$,

Robinson College, CAMBRIDGE U.K.

\title{
Application of fast sensors to microscope mode spatial imaging mass spectrometry
}

\author{
M. Brouard,,$^{a, 1}$ A.J. Johnsen. ${ }^{a}$ A. Nomerotski. ${ }^{b}$ C.S. Slater. ${ }^{a}$ C. Vallance ${ }^{c}$ \\ and W.H. Yuen ${ }^{a}$ \\ ${ }^{a}$ Department of Chemistry, University of Oxford, \\ Physical and Theoretical Laboratory, South Parks Road, Oxford, OX1 3QZ, U.K. \\ ${ }^{b}$ Department of Physics, Denys Wilkinson Building, \\ Keble Road, Oxford, OX1 3RH, U.K. \\ ${ }^{c}$ Department of Chemistry, University of Oxford, \\ Chemistry Research Laboratory, 12 Mansfield Road, Oxford, OX1 3TA, U.K. \\ E-mail: mark.brouard@chem.ox.ac.uk
}

ABSTRACT: The application of fast detectors to microscope mode spatial imaging mass spectrometry, in which the two-dimensional distributions of particular ion masses over a sample are projected onto a two-dimensional detector, allows the imaging of all mass peaks desorbed from a sample on each time-of-flight cycle. Detecting all fragments per duty cycle means that fewer ionisation and acquisition cycles are required for a full set of data, leading to a reduction in overall detection time, amount of sample required, and sample degradation. Results from a proof-of-concept experiment, in which a fast frame transfer charge-coupled device camera (CCD) with $10 \mathrm{~ns}$ time resolution was coupled to a modified velocity-mapped imaging apparatus, are presented.

KEYWORDS: Pixelated detectors and associated VLSI electronics; Instrumentation and methods for time-of-flight (TOF) spectroscopy; Mass spectrometers

\footnotetext{
${ }^{1}$ Corresponding author.
} 


\section{Contents}

1 Introduction 1

2 Experimental 2

3 Results and discussion $\quad 4$

3.1 Results 4

3.2 Resolution determination and aberrations 4

4 Conclusions 6

\section{Introduction}

High-speed detectors, with the capability to record rapid events in real time, have a wide variety of uses [1] and great potential [2], particularly for the recording of non-repeatable events. Microscope-mode spatial imaging mass spectrometry is one particular field in which fast detectors provide numerous advantages over existing sensors. Spatial imaging mass spectrometry determines the two-dimensional distribution of molecules and molecular masses over a sample, with the fragment mass providing species selectivity. Its methods and applications were recently reviewed by Chughtai and Heeren [3]. In microscope-mode spatial imaging mass spectrometry, an area of sample surface is laser-desorbed and ionised, and the distribution of desorbed ions is projected onto a two-dimensional detector by a carefully-tuned electric field generated by the ion optics of the apparatus. Generally, the masses are separated according to their time-of-flight to the detector. Using a standard detection setup, consisting of a pair of microchannel plates, a phosphor, and a conventional CCD camera, a separate experiment is required in order to record the spatial distribution for ions of each individual mass, and acquiring a complete data set for a sample yielding several masses therefore requires multiple data acquisition cycles. Additionally, prior knowledge of the masses of fragments produced is needed in order to determine the appropriate time of flight at which to collect the image.

The application of high-speed image sensors can remove these limitations. The key limitation in the standard setup is the frame length and readout speed of the CCD camera. An effective exposure time on the order of tens of nanoseconds may be achieved through pulsing either the microchannel plates or an image intensifier placed in front of the camera, but the readout speed limits the repetition rate for such a single-mass measurement to a few tens of Hz. In contrast, a sensor with a sufficiently high frame rate allows the collection of all mass peaks from each duty cycle. A complete data set, including images for all mass peaks, may then be acquired in significantly less time, while exposing the sample to fewer laser shots than when a conventional (slow) camera is used. The reduction of time required for a complete set is advantageous in terms of convenience and in limiting ablation and ionisation damage to the samples. Additionally, some biological samples 


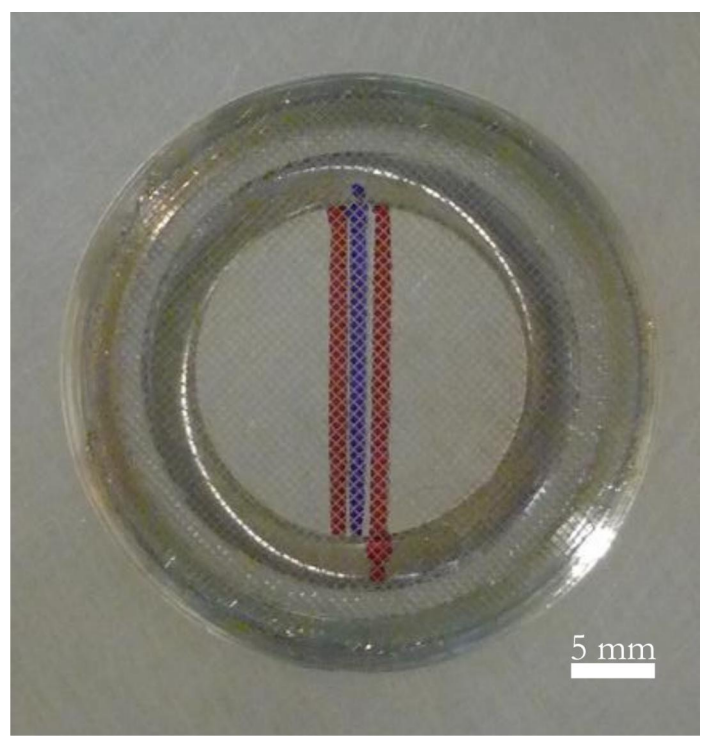

Figure 1. A close-up image of the sample area on the mount. The sample consists of red and blue dyes covered by a nickel mesh.

degrade quickly at room temperatures required for the running of mass spectrometric apparatus [4]. The real-time collection capabilities of fast detectors can also complement the label-free nature of spatial imaging mass spectrometry: by collecting images over the whole period of the cycle after desorption, no prior knowledge of the fragment masses produced is needed.

The feasibility of combining a fast pixel sensor detector with spatial imaging mass spectrometry in order to collect images for multiple mass peaks per duty cycle has been demonstrated here with a proof-of-concept experiment. Although fast detectors have previously been used on a spatial imaging mass spectrometry experiment [5], the speed of the detector assembly was not sufficient to collect ion distributions of multiple masses in each cycle of the experiment. Here, we report proofof-concept measurements in which a sufficiently fast CCD camera (DALSA Corporation) [6] was coupled to a modified velocity-map ion imaging apparatus in order to collect the spatially-resolved distributions of multiple mass fragments per duty cycle.

\section{Experimental}

The experimental apparatus used in this work was an adapted conventional velocity-map [7] ion imaging [8] instrument. The apparatus has been described in detail previously, both with [9] and without $[10,11]$ the fast-framing camera, and only the most recent modifications to the apparatus shall be described here.

A total of four electrostatic lenses, comprised of a repeller plate and three aperture electrodes, were originally used in the ion optics assembly for velocity mapping. These were set up to act as an emission zoom lens [12] by adjusting the potentials of the electrodes. For the work reported here, the repeller was modified to act as a sample mount. The modified electrode consists of a 2 mm-thick stainless steel plate with two circular grooves cut into the surface. A variety of samples were used on different experimental runs. Matrix materials commonly used in matrix-assisted 


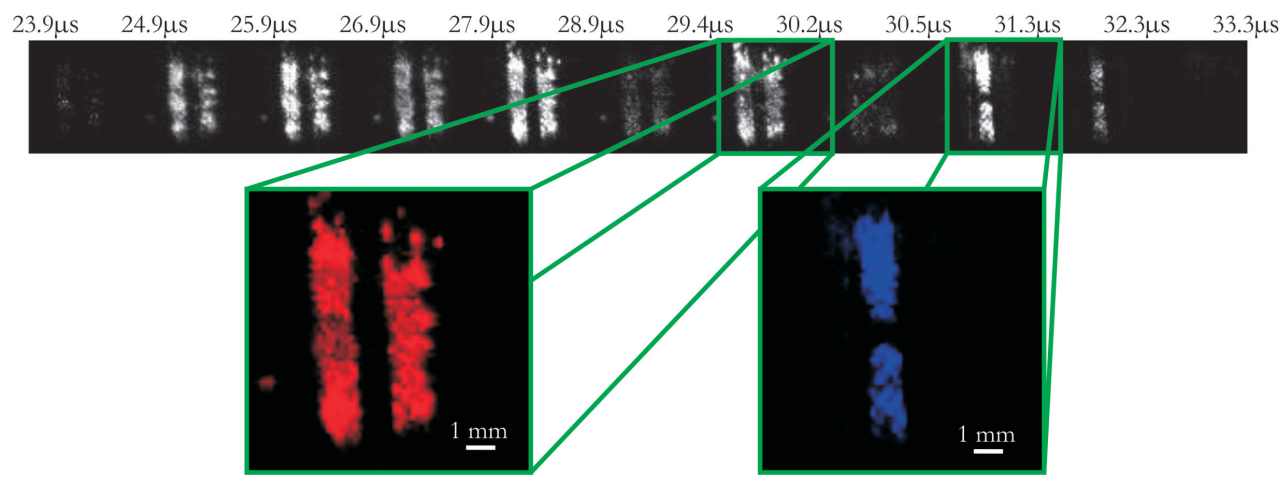

Figure 2. Image of sample shown in figure 1 taken with fast-frame camera. The time between irradiation and the start and end of exposure is indicated. The main mass peaks are expanded and coloured to illustrate the difference in distribution with mass.

laser desorption/ionisation (MALDI) were used as samples: sinapinic acid, $\alpha$-cyano-4-hydroxy cinnamic acid, and di-hydroxy benzoic acid were successfully imaged. Each matrix was made up to $10 \mu \mathrm{g} / \mathrm{ml}$ in acetonitrile and 0.1 (aq.) tri-fluoro-acetic acid. The samples presented here consisted of a mixture of pigments (Staedtler Lumocolour), which produced mass peaks at 478.32 Da (blue pigment), and $385.26 \mathrm{Da}$ and 443.24 $\mathrm{Da}$ (red pigment) when analysed with commercial electrospray ionisation mass spectrometry. A key advantage of using these brightly coloured pigments in our proof-of-concept measurements is the straightforward manner in which the images obtained using the imaging mass spectrometry technique may be compared with photographic images of the prepared samples. The sample was applied to the surface of the mount within the grooves in the desired pattern and air dried. An electro-formed nickel mesh was placed over the sample as a mask for determining resolution. The mesh was stretched taught with an o-ring in the outer groove and attached to the plate with cyanoacrylate glue. The inner groove prevented the glue from flowing onto the sample. The mesh had $74 \%$ transmittance, square holes of $430 \mu \mathrm{m} \times 430$ $\mu \mathrm{m}$ and a pitch of $500 \mu \mathrm{m}$. A photograph of the prepared sample plate is shown in figure 1 . The sample was desorbed using a pulse from an Nd:YAG laser (Continuum Powerlite 8010), which had been attenuated to yield a pulse energy of approximately $20 \mathrm{~mJ}$ at the sample. The laser beam was defocussed using one spherical and one cylindrical lens in order to irradiate the entire sample area. Images were acquired using both the fast-framing camera and a conventional intensified CCD camera (Photonic Science). The framing camera is capable of acquiring and storing 16 frames on chip, with user-programmable exposures for each frame in the range from $10 \mathrm{~ns}$ to $1.25 \mu \mathrm{s}$. The exposures were programmed in order to capture all mass peaks of interest on every TOF cycle. However, the image sensor has only a low spatial resolution of $64 \times 64$ pixels, with a pixel pitch of $100 \mu \mathrm{m}$. Multiple mass acquisition was precluded by the slow frame readout for the intensified $\mathrm{CCD}$, which had to be gated to acquire a single mass per TOF, but this had a much higher resolution of $576 \times 768$ pixels. The mass spectrum was also collected simultaneously in both of these cases from the response of the MCPs. 


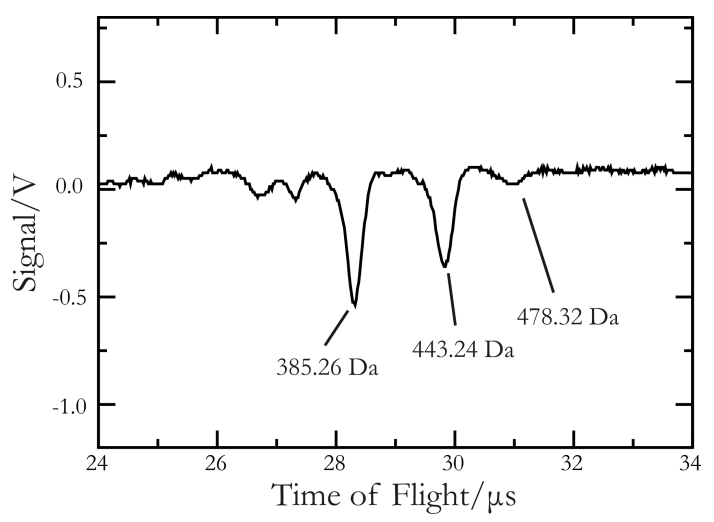

Figure 3. Time of flight acquired from the voltage drop across the MCPs. Masses of the mass peaks are indicated. The FWHM is approximately $300 \mathrm{~ns}$.

\section{Results and discussion}

\subsection{Results}

A representative set of images obtained using the fast frame-transfer camera is shown in figure 2 . Note that this figure shows a subset of the full 16 images acquired by the camera on each duty cycle; the last few frames have not been shown as no further mass peaks were detected at longer times of flight. The images shown are a sum over 200 time-of-flight cycles. The spatial resolution of the $64 \times 64$ pixel sensor is just sufficient to resolve the grid pattern of the nickel mesh. The time labels between each frame indicated the time at which the frame exposure was started and ended, relative to the desorption laser pulse. Exposures were chosen to correspond to particular peaks in the mass spectrum (figure 3), and this data, together with the photograph of the sample in figure 1, allows unambiguous identification of the mass peaks and their spatial origins. The spatial distribution of the image beginning at $30.5 \mu$ s corresponds to that of the blue pigment seen in figure 1 and the time-of-flight matches that shown for the blue pigment in figure 3 . The frames starting from $27.9 \mu \mathrm{s}$ and $29.4 \mu \mathrm{s}$ can be identified from their distributions as originating from the red pigment, and again correspond to the appropriate peaks seen in the time of flight. Additionally, earlier peaks visible in the image which do not correspond to strong peaks in the mass spectrum can be assigned from the spatial distribution to fragmentation products of the red pigment. Three images corresponding to the largest mass peaks have been enlarged in the inset to figure 2 .

\subsection{Resolution determination and aberrations}

Based on the time of flight spectrum in figure 3, the peaks have a maximum width of $700 \mathrm{~ns}$, and a full width half maximum (FWHM) of approximately $300 \mathrm{~ns}$, corresponding to a mass resolution of approximately $5 \mathrm{Da}$ at this mass range. This is partly due to the time resolution of the detection technique: the time of flight is measured from the drop in voltage across the MCPs, and the recovery time is determined by the capacitance of the MCPs, as well as their power supplies. However, peaks have been measured on separate velocity-map imaging experiments on this detector with FWHM of less than $80 \mathrm{~ns}$, and so this should not be the limiting factor of the resolution. Other major factors are the spherical and chromatic aberrations induced by the use of simple aperture 


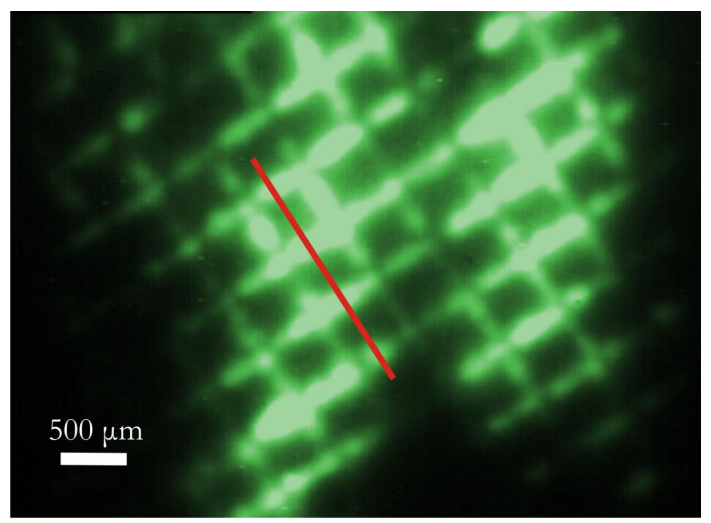

Figure 4. Image collected for determination of spatial resolution. The intensity profile was taken along the red line indicated.

electrodes [12], similar to the incomplete pancaking observed in velocity-map imaging [7]. Simulations were conducted using the SIMION 8.0 [13] ion trajectory modelling package to investigate the extent of these aberrations. The simulation was set up using the same ion lens dimensions and potentials employed in the experiment, and trajectories were calculated for particles of 443.24 Da, corresponding to the mass of the red pigment. Speeds of $750 \mathrm{~ms}^{-1}$, consistent with the values recently described by Knochenmuss et al. [14], were assigned to the particles in a random direction away from the sample plate. The upper limit to the time spread was $700 \mathrm{~ns}$, close to that seen in the time of flight profile. A reflectron could be introduced [15] to improve the mass resolution.

The spatial resolution that could be attained using this apparatus without being limited by the low pixel resolution of the DALSA camera was determined by recording images with the conventional intensified CCD camera. An image of the grid alone, coated with pigment, is shown in figure 4. An intensity profile was taken along the line indicated on this image and the spatial resolution was found by a Gaussian fit to the intensity peaks corresponding to the grid wires to be on the order of $100 \mu \mathrm{m}$. A number of possible factors should be considered as sources of aberration in the images. The apparatus has a magnification factor of 19.5 from the sample to the detector. Any resolution limitation on the detector may therefore be converted to sample resolution by dividing by 19.5. The pores of the MCPs used have a pitch of $32 \mu \mathrm{m}$, corresponding to $1.6 \mu \mathrm{m}$ on the sample. The electron cloud footprint is $1.5 \mathrm{~mm}$ on the phosphor, $77 \mu \mathrm{m}$ on the sample. The entire active area (75 $\mathrm{mm}$ diameter) of the $\mathrm{MCP} /$ phosphor screen detector is projected onto the detection area of the camera for maximum field of view; image acquisition effectively bins the image into a grid equal to the pixel resolution of the camera used. Hence, the fast camera divided the image into $64 \times 64$ squares, with one pixel corresponding to $56 \mu \mathrm{m}$ per pixel on the sample, while the higher resolution camera images $6.2 \mu \mathrm{m}$ at the sample per pixel. Finally, the spherical aberrations and space charge effects were examined using the same ion lens model in SIMION 8.0 as was used to examine the time resolution. A point source was found to yield a spread of impacts with a radius of $2.1 \mathrm{~mm}$ on the detector, corresponding to $108 \mu \mathrm{m}$ on the sample.

Improving the resolution of the apparatus will require the known sources of aberration to be addressed. The pore size of the MCPs is negligible in comparison to the other factors, but the effect of the finite size of the electron cloud emitted from the back face of the MCPs can be re- 
duced using centroiding and event counting [16] procedures, as well as the use of a greater bias between the MCPs and the screen. The aberrations in cylindrically symmetric ion optics stem from the difference in path of non-paraxial trajectories, typical of particles with high velocity perpendicular to the time of flight axis. Removing these by placing an aperture at the velocity mapping (back focal) plane should limit the effects of these aberrations and improve the time resolution. The developments in the use of fast CMOS detectors such as TimePix [17] and PImMS [18] for imaging provide opportunities to use higher resolution detectors, decreasing the resolution lost by the coarseness of the camera. Finally, increased overall magnification would decrease the effects of the electron footprint and pixel size, and is of course generally desirable. Possible methods for improved magnification include the extension of the flight path, perhaps through the use of a reflectron, and the introduction of magnifying ion lenses.

\section{Conclusions}

Spatial imaging mass spectrometry has been demonstrated using a conventional velocity-map imaging apparatus and a fast frame-transfer camera CCD camera. Only minor modifications to a standard velocity-map imaging setup are required in order to convert it to a low-magnification mass spectrometric microscope. The resolution of this microscope is mainly limited by the aberrations stemming from the use of simple aperture electrodes and the low resolution of the detector, but may be reduced by modifications to the ion optics and the use of higher-resolution fast detectors that are or will soon be available.

\section{Acknowledgments}

The support of the U.K. Royal Society and EPSRC via Programme Grant No. EP/G00224X/1 (MB and CV), EU grant FP7 ITN “ICONIC" (MB and CV), ERC grant 200733 "ImageMS" (CV) and a "proof of concept" grant from ISIS Innovation Ltd. (MB, CV and AN) are gratefully acknowledged. This work is subject to a patent application by ISIS Innovations Ltd. (UK patent number 0724295.1; international patent PCT/GB2008/004085).

\section{References}

[1] D.V.T. Son et al., Toward 100 Mega-frames per second: design of an ultimate ultra-high-speed image sensor, Sensors 10 (2010) 16.

[2] A.R. Faruqi and R. Henderson., Electronic detectors for electron microscopy, Curr. Opin. Struct. Bio. 17 (2007) 549.

[3] K. Chughtai and R.M.A. Heeren, Mass spectrometric imaging for biomedical tissue analysis, Chem. Rev. 110 (2010) 3237.

[4] R.J.A. Goodwin, S.R. Pennington and A.R. Pitt, Protein and peptides in pictures: imaging with MALDI mass spectrometry, Proteomics 8 (2008) 3477

[5] S.L. Luxembourg, T.H. Mize, L.A. McDonnell and R.M.A. Heeren, High-spatial resolution mass spectrometric imaging of peptide and protein distributions on a surface, Anal. Chem. 76 (2004) 5339. 
[6] L. Lazovsky, D. Cismas, G. Allan and D. Given, CCD sensor and camera for 100 Mfps burst frame rate image capture, Proc. SPIE 5787 (2005) 184;

Dalsa, http://www.dalsa.com.

[7] A.T.J.B. Eppink and D.H. Parker, Velocity map imaging of ions and electrons using electrostatic lenses: Application in photoelectron and photofragment ion imaging of molecular oxygen, Rev. Sci. Instrum. 68 (1997) 3477.

[8] D.W. Chandler and P.L. Houston, Two dimensional imaging of state-selected photodissociation products detected by multiphoton ionization, J. Chem. Phys. 87 (1987) 1445.

[9] M. Brouard et al., Velocity map imaging in time of flight mass spectrometry, Rev. Sci. Instrum. 79 (2008) 123115.

[10] M.J. Bass, M. Brouard, A.P. Clark and C. Vallance, Fourier moment analysis of velocity-map ion images, J. Chem. Phys. 117 (2002) 8723.

[11] M. Brouard, R. Cireasa, A.P. Clark, T.J. Preston and C. Vallance, The photodissociation dynamics of $\mathrm{NO}_{2}$ at $308 \mathrm{~nm}$ and of $\mathrm{NO}_{2}$ and $\mathrm{N}_{2} \mathrm{O}_{4}$ at $226 \mathrm{~nm}$, J. Chem. Phys. 124 (2006) 064309.

[12] H. Liebl, Applied charged particle optics, Springer, U.S.A. (2008).

[13] SIMION, http://www.simion.com.

[14] R. Knochenmuss and L.V. Zhigilei, Molecular dynamics simulations of MALDI: laser fluence and pulse width dependence of plume characteristics and consequences for matrix and analyte ionization, J. Mass Spec. 285 (2010) 333.

[15] B.D. Leskiw, M.H. Kim, G.E. Hall and A.G. Suits. Reflectron velocity map ion imaging, Rev. Sci. Instrym. 76 (2005) 104101.

[16] B.Y. Chang et al., Improved two-dimensional product imaging: The real-time ion-counting method, Rev. Sci. Instrum. 69 (1998) 1665.

[17] G. Gademann et al., Velocity map imaging using an in-vacuum pixel detector, Rev. Sci. Instrum. 80 (2009) 103105.

[18] A. Nomerotski et al., Pixel imaging mass spectrometry with fast and intelligent pixel detectors, 2010 JINST 5 C07007. 